\title{
Evidence from the eyes: Threatening postures hold attention
}

\author{
Bobby Azarian $^{1}$ - Elizabeth G. Esser ${ }^{2}$ - Matthew S. Peterson ${ }^{1,2}$
}

Published online: 18 September 2015

(C) Psychonomic Society, Inc. 2015

\begin{abstract}
Efficient detection of threat provides obvious survival advantages and has resulted in a fast and accurate threatdetection system. Although beneficial under normal circumstances, this system may become hypersensitive and cause threat-processing abnormalities. Past research has shown that anxious individuals have difficulty disengaging attention from threatening faces, but it is unknown whether other forms of threatening social stimuli also influence attentional orienting. Much like faces, human body postures are salient social stimuli, because they are informative of one's emotional state and next likely action. Additionally, postures can convey such information in situations in which another's facial expression is not easily visible. Here we investigated whether there is a threat-specific effect for high-anxious individuals, by measuring the time that it takes the eyes to leave the attended stimulus, a task-irrelevant body posture. The results showed that relative to nonthreating postures, threat-related postures hold attention in anxious individuals, providing further evidence of an anxiety-related attentional bias for threatening information. This is the first study to demonstrate that attentional disengagement from threatening postures is affected by emotional valence in those reporting anxiety.
\end{abstract}

Keywords Attention $\cdot$ Threat $\cdot$ Postures $\cdot$ Anxiety $\cdot$ Eye movements

\section{Bobby Azarian}

sazarian@gmu.edu

1 Neuroscience Program, George Mason University, 4400 University Drive MS 3F5, Fairfax, VA 22030, USA

2 Department of Psychology, George Mason University, Fairfax, VA, USA
Critical to an organism's survival is the ability to efficiently detect threat in the environment. Consequently, an attentional system specialized for the rapid detection of threat has been shaped over the course of our evolutionary history. The results from numerous studies using visual search tasks have showed automatic orienting of attention to threatening stimuli such as angry faces (Eastwood, Smilek, \& Merikle, 2001; Fox et al., 2000; Öhman, Lundqvist, \& Esteves, 2001), snakes, and spiders (Öhman, Flykt, \& Esteves, 2001), allowing for quicker coping responses to harmful agents.

In addition to capturing attention, threatening information has been shown to delay attention from leaving the attended threat. This delay in disengagement has been commonly referred to as "attentional dwelling" (Fox, Russo, Bowles, \& Dutton, 2001; Fox, Russo, \& Dutton, 2002) and has been shown to occur in response to threat-related words (Fox et al., 2001), threatening pictures (Yiend \& Mathews, 2001), and angry faces (Fox et al., 2001; Fox et al., 2002) in individuals with high levels of selfreported anxiety. Common paradigms used in such research include various modifications of the classic spatial-cueing task (Posner, 1980), in which participants must disengage attention from a spatially distinct psychophysical stimulus in order to locate or identify a subsequent peripheral target. If a specific stimulus characteristic, such as being threat-related, does in fact delay attentional disengagement, then the average time required to respond to targets following threatening stimuli should be longer than the average response time to targets presented after neutral or nonthreatening stimuli.

Past investigations of attentional disengagement have primarily relied on reactions times from buttonpress responses to peripheral targets, which can only allow inferences of disengagement and may be insufficiently sensitive to subtle effects. A recent study by Belopolsky, Devue, and Theeuwes (2011) showed delayed disengagement from angry faces in the general population by using eyetracking, a measure with 
greater sensitivity than previously used methods. Given the tight coupling of covert attention and saccades (Belopolsky \& Theeuwes, 2009; Deubel \& Schneider, 1996), along with the fact that an eye movement first requires a covert shift of attention to the point of destination (Deubel \& Schneider, 1996; Peterson, Kramer, \& Irwin, 2004), oculomotor disengagement provides a more direct measure of the time of attentional disengagement (Brockmole \& Boot, 2009). In light of these points, for our investigation we also employed eyetracking to more directly measure attentional disengagement, by determining the time it takes an individual to initiate a saccade away from an engaged posture stimulus. We adopted a previously used design (Belopolsky et al., 2011; Georgiou et al., 2005) that includes a central posture display followed by a peripheral target letter that may appear either to the left or to the right of the stimulus, 100 or $500 \mathrm{~ms}$ after stimulus onset. This central display design is superior to other commonly used tasks (e.g., the exogenous spatial-cueing task or the dot-probe task) that require one first to orient toward the social cue. Since in our task the social cue was already at fixation, we eliminated the need to shift attention toward it. Thus, the present study design and methodology offer a number of advantages over traditional approaches.

At present, there is no evidence as to whether differences in attentional disengagement occur only in response to angry faces or may generalize to other forms of social stimuli expressing anger - in particular, direct-facing angry body postures. Past research has shown that faces are unique stimuli, often referred to as "special" in that they receive preferential visual processing (Farah, Wilson, Drain, \& Tanaka, 1998) carried out by specialized neural modules such as the fusiform face area (Kanwisher, McDermott, \& Chun, 1997). However, Gilbert, Martin, and Coulson (2011) demonstrated that threatening postures are detected more readily than nonthreatening postures during visual search, suggesting that delayed disengagement might generalize to other social stimuli, such as angry postures. Interestingly, Gilbert et al. also reported delayed disengagement, but these findings could be attributed to the threatening posture distractors capturing attention more than nonthreatening distractors, thus delaying search times. Their indirect measure, along with the use of averted rather than direct posture stimuli, further obscures the disengagement issue, since averted angry postures have been shown to cue attention rather than to delay it (Azarian, Esser, \& Peterson, 2015). In addition, averted heads (Langton \& Bruce, 1999), directional hand gestures (Langton, Watt, \& Bruce, 2000), and threatening faces with averted eye gaze (Fox, Mathews, Calder, \& Yiend, 2007; Mathews, Fox, Yiend, \& Calder, 2003) have also been shown to cue attention in the signaled direction. To avoid this potential confound, we used only direct-facing postures.

In addition, although facial expressions can clearly convey the emotional state of an individual, posture expressions may be equally informative of emotion, and to lesser extents, identity and gender. In some instances, posture expression may be a better predictor of another individual's next likely action, since the body must be oriented in the appropriate direction before a movement can be carried out. For example, an aggressive direct-facing posture can be a stronger signal of immediate danger than an angry facial expression. Additionally, a body posture may be the only social cue available in visually degraded situations in which the face is occluded, or when viewing another individual from a long distance.

Along with the consistent finding that angry facial expressions delay disengagement in high-anxious individuals, fearful faces have shown similar effects (Georgiou et al., 2005), indicating that such behavior may be more generally related to threat than to a single expressed emotion. In fact, an experiment by Bannerman, Milders, and Sahraie (2010) that reported attentional capture by fearful postures also revealed impaired disengagement in an exogenous cueing task at the shortest cue duration $(20 \mathrm{~ms})$. However, their study only looked at individuals reporting normal levels of trait anxiety, potentially missing any information regarding the modulatory role of anxiety that has so often been seen in prior investigations. Furthermore, no other nonneutral expressions were tested, leaving open the possibility that simple emotional valence is what holds attention, especially considering that the participants were not anxious. To ensure that any effects found were specific to threat, we included happy postures in addition to neutral ones. On the basis of the aforementioned findings, we predicted that both angry and fearful postures would hold the eyes.

Research suggests that emotional body postures are salient social stimuli that have the power to influence how individuals perceive faces. An eyetracking study by Aviezer et al. (2008) showed that the perception of a facial expression can be categorically altered if it is presented in conjunction with a conflicting emotional body posture. For example, a facial expression conveying disgust might be perceived as an angry expression if the body posture is expressing anger. Similarly, a 2014 study by Van den Stock and de Gelder showed that taskirrelevant emotional body postures could influence the perception of facial identity. The salience and significance of emotional postures has been further illustrated by the fact that they are processed and categorized correctly even without conscious perception. When angry postures are presented to those with clinical blindness caused by striate cortex lesions, they elicit activation of subcortical brain areas including the superior colliculus and bilateral pulvinar (Van den Stock et al., 2011). Such properties of emotional postures highlight the need to further study their influence on cognition and, in the case of the present experiment, visual attention.

The present study is a logical extension of previous research. If other forms of social stimuli beyond faces, such as threat-related postures, have been shown to affect attentional 
disengagement in anxious individuals, it is presumable that attentional dwelling is more frequent and pervasive than has previously been imagined. Whether this automatic behavior increases the anxious state through dwelling on negative aspects of the environment (Fox et al., 2002) or is adaptive, allowing for more elaborate processing in order to execute an appropriate defense (Belopolsky et al., 2011), has yet to be seen conclusively. If eyetracking were to reveal a disengagement effect in response to threatening relative to nonthreatening postures only in high-anxious individuals, this may implicate a causal role for the anxious state in maintaining attention. In particular, we might expect to find a disengagement effect at short stimulus onset asynchronies (SOAs), suggesting that the effect is due to automatic rather than intentional processes.

\section{Method}

\section{Participants}

A group of 22 undergraduates (14 female, eight male) with ages ranging from 18 to 27 years (mean age $=20$ ) were recruited from the George Mason University community. The sample size was chosen on the basis of previous oculomotor disengagement studies. Eleven of the participants had trait anxiety scores of 45 or above on the State-Trait Anxiety Inventory (STAI-T) and were assigned to the high-anxiety group, whereas 11 scored 35 or below and were assigned to the low-anxiety group. A threshold of 39-40 has been suggested for clinically significant scores (Julian, 2011), and the highest score in our low-trait group $(34, M=29.9)$ and the lowest score of our high-trait group $(47, M=54.3)$ both fell safely on either side of that threshold.

All participants had normal or corrected-to-normal vision.

\section{Materials and apparatus}

Posture stimuli were created depicting fearful, angry, happy, and neutral expressions. In a pilot study, 20 participants were asked to choose which of the four emotions was being expressed by each posture presented at random. The results confirmed that all were correctly recognized above $80 \%$. Three male actors each provided three versions of each of the four different emotions, yielding 36 unique averted posture stimuli. The choice of a male gender for the actors was based on previous findings showing that such stimuli evoke greater arousal when fear and anger are expressed (Kret, Pichon, Grèzes, \& de Gelder, 2011). Grayscale images had the faces blurred and were presented in the center of screen, subtending $7^{\circ} \times 19^{\circ}$ of visual angle at a viewing distance of $60 \mathrm{~cm}$. Target dots subtended $1^{\circ}$ of visual angle and were presented $10^{\circ}$ to either the left or right of fixation. All stimuli were presented on a MacPro $(2 \times 2 \mathrm{GHz}$ Dual-Core Intel
Xenon) equipped with a 20 -in. CRT monitor operating at $75 \mathrm{~Hz}$ with a resolution of $1,024 \times 768$. This computer was networked to a Dell Pentium 4 processor that collected eyetracking data in conjunction with an EyeLink 2 eyetracker (SR Research, Ontario, Canada), which sampled at a rate of $250 \mathrm{~Hz}$ with 0.2 spatial resolution. Examples of the stimuli depicting each emotion are displayed in Fig. 1a.

\section{Procedure}

Each trial began with a central fixation circle. The participant pressed the space bar to perform a drift correction, after which a fixation cross was displayed for $1,000 \mathrm{~ms}$, followed by a posture stimulus. The target then appeared in a nonpredictive fashion to the left or the right of the posture either 100 or $500 \mathrm{~ms}$ after its appearance. The target and the posture remained on the screen until a saccade was made or until 2, $000 \mathrm{~ms}$ passed. An example of a trial with an angry posture expression is illustrated in Fig. $1 \mathrm{~b}$.

The saccadic reaction time was defined as the time between target onset and the saccade start time, with the saccade start being defined as when the eyes passed a boundary $2.5^{\circ}$ to the left or the right of fixation. Participants were asked to keep their eyes in the center of the screen until the target appeared, and they were instructed to make an eye movement toward the target as quickly and accurately as possible. If a saccade was made prematurely (crossing a boundary $2.5^{\circ}$ to the left or the right), the message "you moved your eyes too soon" appeared on the screen, and that trial was recycled and randomly inserted later in the experiment. Participants completed one practice block of 12 trials, followed by one experimental block of 288 trials.

\section{Design}

We first carried out two preliminary mixed 2 (Anxiety) $\times 2$ $($ Expression $) \times 2$ (SOA) analyses of variance (ANOVAs) to determine whether significant differences existed between the threat-related expressions (fearful and angry) and between the neutral expressions (neutral and happy). If the analysis showed no significant differences, then the fearful and angry expressions could be collapsed into a threat category, and the neutral and happy expressions could be collapsed into a nonthreat category. We then carried out a 2 (Anxiety: low and high $) \times 2$ (Expression: threat and nonthreat $) \times 2($ SOA: 100 and $500 \mathrm{~ms}$ ) mixed ANOVA with Anxiety as a betweensubjects factor, and the average time taken to initiate a saccade toward the target as the dependent variable.

\section{Results}

Saccade latencies less than $80 \mathrm{~ms}$ or greater than $500 \mathrm{~ms}$ were discarded from the analysis. A 2 (Anxiety Level: high or 
a

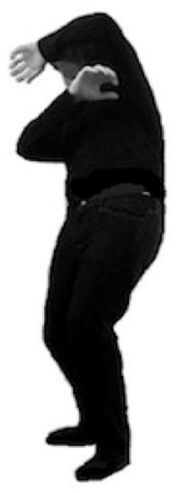

Fear

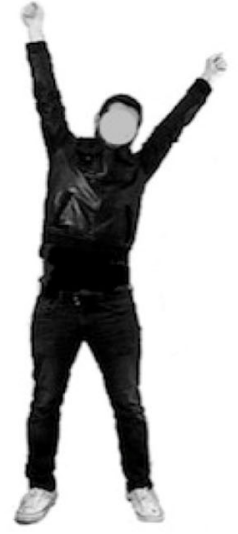

Happy

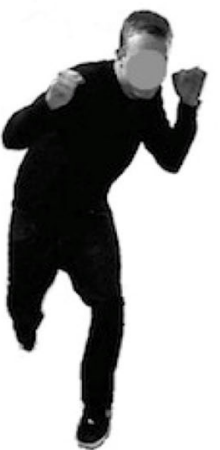

Anger

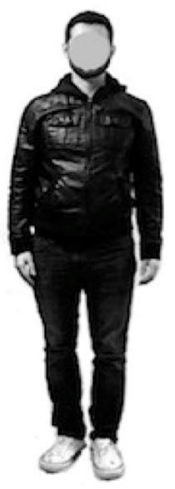

Neutral

b

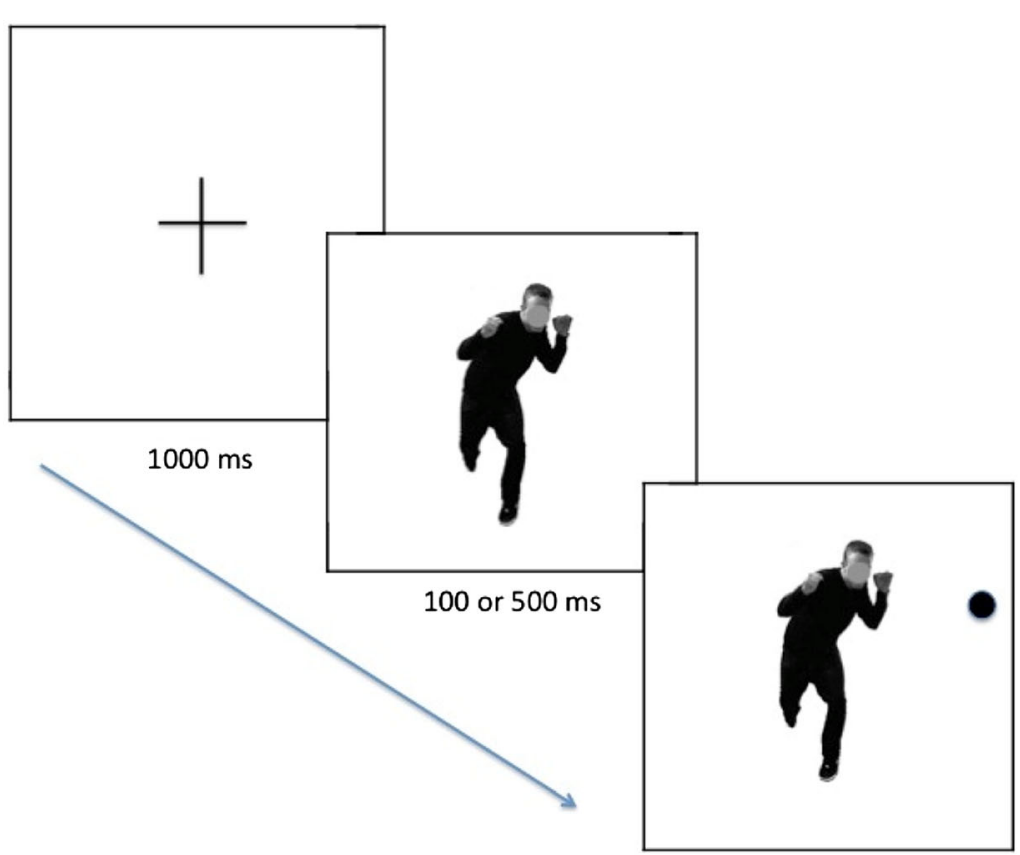

Until Saccade

Fig. 1 a Examples of direct postures expressing each emotional type. b Example of an anger trial (not to scale). Participants were instructed to make a saccade from the posture toward the target dot as quickly and accurately as possible

low) $\times 2$ (Expression: fear or anger) $\times 2$ (SOA: 100 or $500 \mathrm{~ms}$ ) mixed ANOVA with Anxiety as a between-subjects factor showed only a main effect of SOA, $F(1,20)=4.5, p=$ $.047, \eta_{\mathrm{p}}{ }^{2}=.183$, with no significant main effects of emotion, $F(1,20)=1.1, p=.31, \eta_{\mathrm{p}}{ }^{2}=.051$, or anxiety, $F(1,20)<.01, p=$ $.96, \eta_{\mathrm{p}}{ }^{2}<.001$. A 2 (Anxiety: high or low) $\times 2$ (Expression: neutral or happy) $\times 2$ (SOA: 100 or $500 \mathrm{~ms})$ mixed ANOVA yielded no significant main effects or interactions. Thus, fear and anger were collapsed together (threat), and neutral and happy were also collapsed (nonthreat), for each individual SOA. Tests for normality, using the Shapiro-Wilk test, indicated that the data were not statistically different from normal (lowest $p=.08$ ).
We carried out a 2 (Anxiety Level: high or low) $\times 2$ (Expression: threat or nonthreat) $\times 2$ (SOA: 100 or $500 \mathrm{~ms}$ ) mixed ANOVA with Anxiety as a between-subjects factor, and this yielded no significant main effects of emotion, $F(1,20)=$ $0.37, p=.56, \eta_{\mathrm{p}}{ }^{2}=.018$; anxiety level, $F(1,20)=0.11, p=.74$, $\eta_{\mathrm{p}}{ }^{2}=.006$; or SOA, $F(1,20)=3.4, p=.08, \eta_{\mathrm{p}}{ }^{2}=.146$. The only significant effect was an interaction between emotion, anxiety, and SOA, $F(1,20)=4.5, p=.046, \eta_{\mathrm{p}}{ }^{2}=.184$. Pairwise comparisons of threat versus nonthreat for the four SOA-and-anxietylevel combinations revealed an effect only for anxious individuals at the 100-ms SOA: Saccade RTs following threat-related postures were slower than RTs following non-threat-related postures, $t(10)=4.0, p=.003$ (Bonferroni alpha $=.0125), \eta_{\mathrm{p}}{ }^{2}=.61$. 


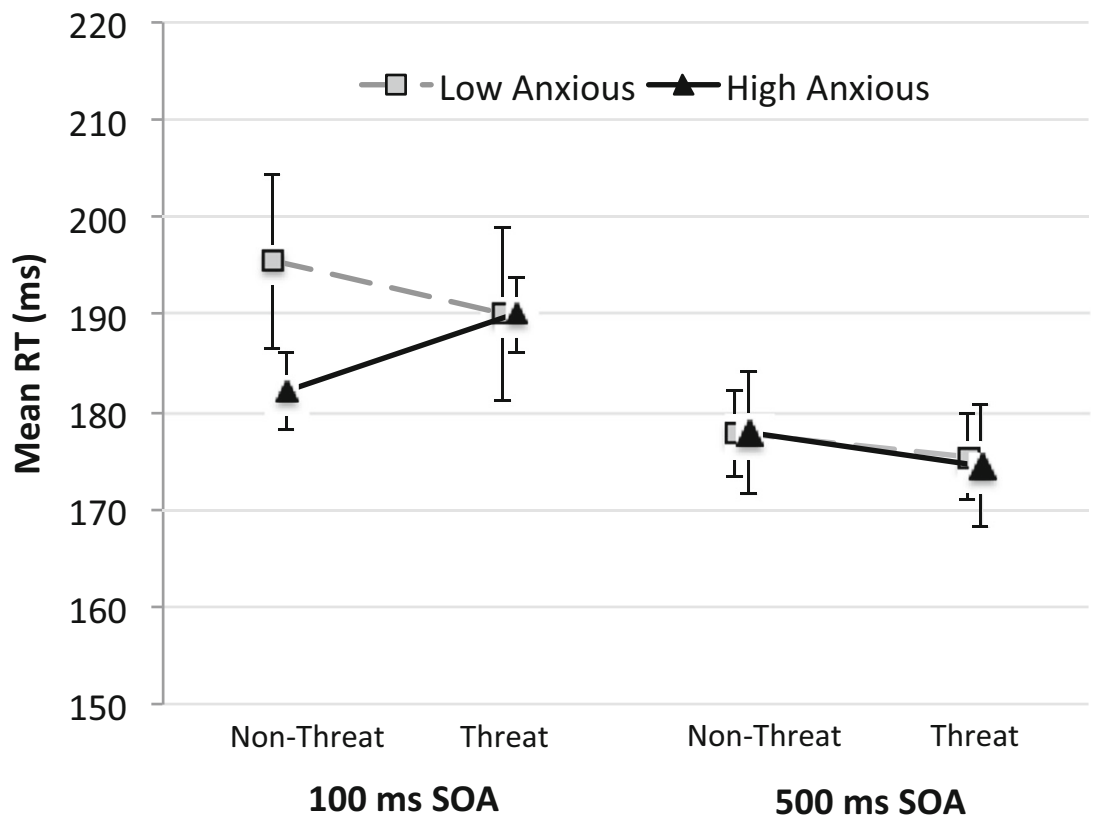

Fig. 2 Saccade latencies (in milliseconds) with emotions collapsed into threat and nonthreat categories and divided between anxiety groups. Error bars represent the $95 \%$ confidence intervals for the paired threat versus nonthreat $t$ tests

This is represented in Fig. 2. No other comparisons reached significance [highest $t(10)=1.7$, lowest $p=.27$ ].

The results above indicate a significant threat-related effect at short SOAs for the high-anxiety group, as can be seen in Fig. 3. However, Fig. 2 suggests that this effect might be due to faster disengagement from nonthreat for the high-anxious relative to the lowanxious group. We performed post-hoc tests comparing the groups' saccadic reaction times for each threat at each SOA. None of the tests reached statistical significance [highest $t(20)=0.87$, lowest $p=.39$ ], indicating

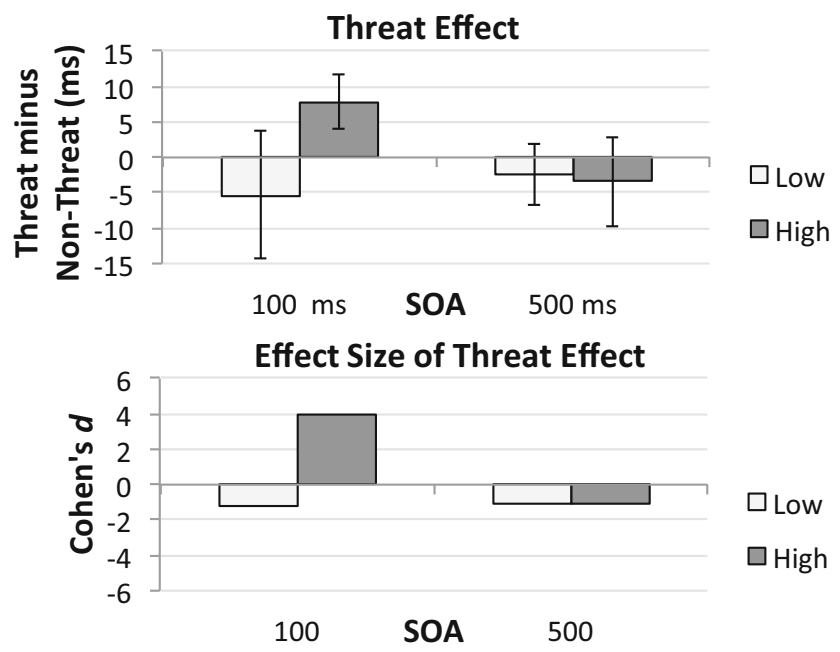

Fig. 3 Threat effect (threat vs. nonthreat) effect sizes as a function of trait anxiety and SOA. Error bars represent the $95 \%$ confidence intervals no between-group differences for any of the threats at any of the SOAs.

\section{Discussion}

Our results are straightforward and in agreement with the previous literature: Once attended, threatening postures automatically affect attentional disengagement at short SOAs, but only in those reporting high levels of trait anxiety. When presented with a posture cue at central fixation, participants took longer to initiate a saccade in the direction of a peripheral target when the cue expressed fear or anger relative to happy or neutral postures. This inability to immediately look away suggests that attentional disengagement is delayed by the threatening information encoded in the posture expression, resulting in an attentional dwell. Tracking the eyes allowed us to closely trace the time course of attentional orienting by identifying the exact moment of oculomotor disengagement. It is important to note that emotion was task-irrelevant, so any orienting differences between expression types can be said to be automatic and involuntary. As expected, this effect was present only at the short SOA $(100 \mathrm{~ms})$ and had disappeared by $500 \mathrm{~ms}$, indicating its automatic nature.

These findings expand upon previous research by demonstrating that increased dwell times occur not only in response to threatening faces (Fox et al., 2001; Fox et al., 2002), but to social threat more generally, as was displayed here with directfacing threat-related postures. This suggests that threatening postures and faces influence perception and attention 
similarly. Both receive preferential processing, in that they not only capture attention faster (Gilbert et al., 2011; Öhman, Lundqvist, \& Esteves, 2001), but also hold attention longer, relative to nonthreat. Thus, both angry and fearful postures add to a variety of stimuli that contribute to a robust threatrelated attentional bias (Yiend, 2010).

It has been proposed that the tendency to reflexively dwell on threat may be a form of adaptive behavior (Belopolsky et al., 2011), whereby more time is allotted for processing the threat and developing an appropriate defense strategy. This stands in contrast to the hypothesis that prolonged dwelling increases anxiety by keeping cognitive resources placed on the source of threat, over time leading to worry and rumination (Fox et al., 2001; Georgiou et al., 2005). Our results provide support for the latter theory, since deficient disengagement from threat relative to nonthreat was observed only in trait-anxious individuals. Taking into account these findings, it may be that the ability to rapidly disengage attention from threat keeps anxiety low (Fox et al., 2001). Fox and colleagues (2001) have also proposed that this extended dwell time may reflect freezing behavior that we commonly see in animals in response to danger (see LeDoux, 1996). Therefore, it is no surprise that the effect was present at the shorter SOA, since freezing would presumably be a reflexive response. Furthermore, threat in the form of postures may have been a more salient indicator of an approaching danger than facial expressions at an earlier time in our evolutionary history, particularly before spoken language emerged, so that less attentional focus was placed on another's face. Neural support for this comes from the finding that primates have specialized regions for body processing that do not overlap with face processing areas, including the fusiform body area and the extrastriate body area (Peelen, Atkinson, Andersson, \& Vuilleumier, 2007).

If the eyes are automatically held by screen-sized, static, grayscale psychophysical stimuli when threat is displayed, it is tenable that the dynamic and interactive threatening stimuli of real social or confrontational situations modulate disengagement in a similar fashion. As such, involuntary dwelling on negative aspects of the environment may be frequent and excessive in those with anxiety. Additionally, the onscreen stimuli of various forms of violent visual entertainment, such as violent television and video games, may also influence orienting and perhaps hold attention. Given the frequency of such stimuli in modern times, the resulting attentional patterns could keep one routinely mentally occupied with threatening information, placing extra demand on limited attentional resources and putting overall strain on the cognitive system.

Such reasoning is speculative, as were previous interpretations of lab-controlled results, and investigations that explore threat's impact on orienting in real-world environments will be necessary. Whether an appropriate defense response or simply biased negative dwelling, threatening postures hold attention when anxiety is present, and do so in a manner that is beyond one's control. The question of whether the observed threat bias in anxious individuals is an adaptive response aimed at more elaborate threat processing or is simply a negative consequence of the anxious state that causes overfocusing on threat requires further testing. However, the results unequivocally support the notion that threatening postures hold attention when anxiety is present, and do so in a manner that is beyond one's control.

Author note B.A. and M.S.P. developed the study concept and contributed to the design. Testing and data collection were performed by B.A. and E.G.E. Then, B.A., E.G.E., and M.S.P. performed the data analysis and interpretation, B.A. drafted the manuscript, and M.S.P. provided critical revisions. All authors approved the final version of the manuscript for submission.

\section{References}

Aviezer, H., Hassin, R. R., Ryan, J., Grady, C., Susskind, J., Anderson, A., \& Bentin, S. (2008). Angry, disgusted, or afraid? Studies on the malleability of emotion perception. Psychological Science, 19, 724 732. doi:10.1111/j.1467-9280.2008.02148.x

Azarian, B., Esser, E. G., \& Peterson, M. S. (2015). Watch out! Directional threat-related postures cue attention and the eyes. Cognition and Emotion. doi:10.1080/02699931.2015.1013089

Bannerman, R. L., Milders, M., \& Sahraie, A. (2010). Attentional cueing: Fearful body postures capture attention with saccades. Journal of Vision, 10(5), 23. doi:10.1167/10.5.23

Belopolsky, A. V., Devue, C., \& Theeuwes, J. (2011). Angry faces hold the eyes. Visual Cognition, 19, 27-36. doi:10.1080/13506285.2010. 536186

Belopolsky, A. V., \& Theeuwes, J. (2009). When are attention and saccade preparation dissociated? Psychological Science, 20, 1340 1347. doi:10.1111/j.1467-9280.2009.02445.x

Brockmole, J. R., \& Boot, W. R. (2009). Should I stay or should I go? Attentional disengagement from visually unique and unexpected items at fixation. Journal of Experimental Psychology: Human Perception and Performance, 35, 808-815.

Deubel, H., \& Schneider, W. X. (1996). Saccade target selection and object recognition: Evidence for a common attentional mechanism. Vision Research, 36, 1827-1837. doi:10.1016/0042-6989(95) 00294-4

Eastwood, J. D., Smilek, D., \& Merikle, P. M. (2001). Differential attentional guidance by unattended faces expressing positive and negative emotion. Perception \& Psychophysics, 63, 1004-1013. doi:10. 3758/BF03194519

Farah, M. J., Wilson, K.-D., Drain, M., \& Tanaka, J. N. (1998). What is "special" about face perception? Psychological Review, 105, 482498. doi:10.1037/0033-295X.105.3.482

Fox, E., Lester, V., Russo, R., Bowles, R. J., Pichler, A., \& Dutton, K. (2000). Facial expressions of emotion: Are angry faces detected more efficiently? Cognition and Emotion, 14, 16-92.

Fox, E., Mathews, A., Calder, A., \& Yiend, J. (2007). Anxiety and sensitivity to gaze direction in emotionally expressive faces. Emotion, 7, 478-486.

Fox, E., Russo, R., Bowles, R., \& Dutton, K. (2001). Do threatening stimuli draw or hold visual attention in subclinical anxiety? Journal of Experimental Psychology: General, 130, 681-700. doi: 10.1037/0096-3445.130.4.681 
Fox, E., Russo, R., \& Dutton, K. (2002). Attentional bias for threat: Evidence for delayed disengagement from emotional faces. Cognition and Emotion, 16, 355-379.

Georgiou, G. A., Bleakley, C., Hayward, J., Russo, R., Dutton, K., Eltiti, S., \& Fox, E. (2005). Focusing on fear: Attentional disengagement from emotional faces. Visual Cognition, 12, 145-158.

Gilbert, T., Martin, R., \& Coulson, M. (2011). Attentional biases using the body in the crowd task: Are angry body postures detected more rapidly? Cognition and Emotion, 25, 700-708. doi:10.1080/ 02699931.2010 .495881

Julian, L. J. (2011). Measures of anxiety: State-Trait Anxiety Inventory (STAI), Beck Anxiety Inventory (BAI), and Hospital Anxiety and Depression Scale-Anxiety (HADS-A). Arthritis Care and Research, 63, S467-S472.

Kanwisher, N., McDermott, J., \& Chun, M. M. (1997). The fusiform face area: A module in human extrastriate cortex specialized for face perception. Journal of Neuroscience, 17, 4302-4311.

Kret, M. E., Pichon, S., Grèzes, J., \& de Gelder, B. (2011). Men fear other men most: Gender specific brain activations in perceiving threat from dynamic faces and bodies-An fMRI study. Frontiers in Psychology, 2, 3. doi:10.3389/fpsyg.2011.00003

Langton, S. R. H., \& Bruce, V. (1999). Reflexive visual orienting in response to the social attention of others. Visual Cognition, 6, 541-567.

Langton, S. R. H., Watt, R. J., \& Bruce, V. (2000). Do the eyes have it? Cues to the direction of social attention. Trends in Cognitive Sciences, 4, 50-59. doi:10.1016/S1364-6613(99)01436-9

LeDoux, J. (1996). The emotional brain. New York: Simon \& Schuster.

Mathews, A., Fox, E., Yiend, J., \& Calder, A. (2003). The face of fear: Effects of eye gaze and emotion on visual attention. Visual Cognition, 10, 823-835. doi:10.1080/13506280344000095

Öhman, A., Flykt, A., \& Esteves, F. (2001a). Emotion drives attention: Detecting the snake in the grass. Journal of Experimental
Psychology: General, 130, 466-478. doi:10.1037/0096-3445.130. 3.466

Öhman, A., Lundqvist, D., \& Esteves, F. (2001b). The face in the crowd revisited: A threat advantage with schematic stimuli. Journal of Personality and Social Psychology, 80, 381-396. doi:10.1037/ 0022-3514.80.3.381

Peelen, M. V., Atkinson, A. P., Andersson, F., \& Vuilleumier, P. (2007). Emotional modulation of body-selective visual areas. Social Cognitive and Affective Neuroscience, 2, 274-283. doi:10.1093/ scan $/ \mathrm{nsm} 023$

Peterson, M. S., Kramer, A. F., \& Irwin, D. E. (2004). Covert shifts of attention precede involuntary eye movements. Perception \& Psychophysics, 66, 398-405. doi:10.3758/BF03194888

Posner, M. I. (1980). Orienting of attention. Quarterly Journal of Experimental Psychology, 32, 3-25. doi:10.1080/ 00335558008248231

Van den Stock, J., \& de Gelder, B. (2014). Face identity matching is influenced by emotions conveyed by face and body. Frontiers in Human Neuroscience, 8, 53. doi:10.3389/fnhum.2014.00053

Van den Stock, J., Tamietto, M., Sorger, B., Pichon, S., Grèzes, J., \& de Gelder, B. (2011). Cortico-subcortical visual, somatosensory, and motor activations for perceiving dynamic whole-body emotional expressions with and without striate cortex (V1). Proceedings of the National Academy of Sciences, 108, 16188-16193. doi:10. 1073/pnas. 1107214108

Yiend, J. (2010). The effects of emotion on attention: A review of attentional processing of emotional information. In J. De Houwer \& D. Hermans (Eds.), Cognition and emotion: Reviews of current research and theories (pp. 211-275). New York: Psychology Press.

Yiend, J., \& Mathews, A. (2001). Anxiety and attention to threatening pictures. Quarterly Journal of Experimental Psychology, 54A, 665681. 